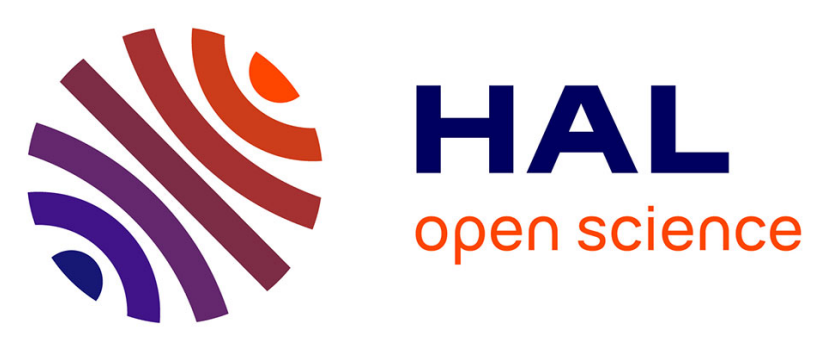

\title{
Asymptotic Finite-Time Ruin Probabilities for a Class of Path-Dependent Heavy-Tailed Claim Amounts Using Poisson Spacings
}

Romain Biard, Claude Lefèvre, Stéphane Loisel, Haikady Nagaraja

\section{- To cite this version:}

Romain Biard, Claude Lefèvre, Stéphane Loisel, Haikady Nagaraja. Asymptotic Finite-Time Ruin Probabilities for a Class of Path-Dependent Heavy-Tailed Claim Amounts Using Poisson Spacings. Applied Stochastic Models in Business and Industry, 2011, 27 (5), pp.503-518. 10.1002/asmb.857. hal-00409418

\section{HAL Id: hal-00409418 https://hal.science/hal-00409418}

Submitted on 7 Aug 2009

HAL is a multi-disciplinary open access archive for the deposit and dissemination of scientific research documents, whether they are published or not. The documents may come from teaching and research institutions in France or abroad, or from public or private research centers.
L'archive ouverte pluridisciplinaire HAL, est destinée au dépôt et à la diffusion de documents scientifiques de niveau recherche, publiés ou non, émanant des établissements d'enseignement et de recherche français ou étrangers, des laboratoires publics ou privés. 


\title{
Asymptotic Finite-Time Ruin Probabilities for a Class of Path-Dependent Heavy-Tailed Claim Amounts Using Poisson Spacings
}

\author{
Romain Biard \\ Université de Lyon, Université Lyon 1, Institut de Science Financière et d'Assurances, \\ 50 Avenue Tony Garnier, F-69007 Lyon, France \\ Ph. : +33437287636, Fax : +33437287632, email : romain.biard@univ-lyon1.fr \\ Claude Lefèvre \\ Université Libre de Bruxelles, Département de Mathématique, Campus de la Plaine \\ C.P. 210, B-1050 Bruxelles, Belgique \\ Ph. : +3226505894, Fax : +3226505867, email : clefevre@ulb.ac.be \\ Stéphane Loisel \\ Université de Lyon, Université Lyon 1, Institut de Science Financière et d'Assurances, \\ 50 Avenue Tony Garnier, F-69007 Lyon, France \\ Ph. : +33437287429, Fax : +33437287632, email : stephane.loisel@univ-lyon1.fr

\section{Haikady N. Nagaraja} \\ Ohio State University, Department of Statistics, 440H Cockins Hall, 1958 Neil Avenue, \\ Columbus $\mathrm{OH} 43210-1247$, U.S.A. \\ Ph. : +16142926072, Fax : +16142922096, email ; hnn@stat.osu.edu
}

\begin{abstract}
Résumé
In the compound Poisson risk model, several strong hypotheses may be found too restrictive to describe accurately the evolution of the reserves of an insurance company. This is especially true for a company that faces natural disaster risks like earthquake or flooding. For such risks, claim amounts are often inter-dependent and they may also depend on the history of the natural phenomenon. The present paper is concerned with a situation of this kind where each claim amount depends on the previous interclaim arrival time, or on past interclaim arrival times in a more complex way. Our main purpose is to evaluate, for large initial reserves, the asymptotic finite-time ruin probabilities of the company when the claim sizes have a heavy-tailed distribution. The approach is based more particularly on the analysis of spacings in a conditioned Poisson process.
\end{abstract}

Keywords : Risk process, finite-time ruin probabilities, asymptotic approximation for large initial reserves, path-dependent claim amount, heavy-tailed claim amount, Poisson spacings.

\section{Introduction}

The compound Poisson risk model is the central model proposed in insurance theory. An abundant literature has been devoted to its analysis and applications. The 
reader is referred e.g. to the comprehensive books by Rolski et al. (1999), Asmussen (2000) and Kaas et al. (2001). It is well recognized, however, that the simplifying hypotheses at the basis of the model may be too restrictive for certain insurance coverages, especially with rare but extreme risks like earthquake or flooding. The present paper is concerned with situations of that kind.

\subsection{Framework and motivations}

Let us begin by recalling the model in its standard version. The reserves of the company, $\{R(t), t \geq 0\}$, are given by

$$
R(t)=u+c t-S(t), \quad t \geq 0,
$$

where $u \geq 0$ is the amount of initial reserves, $c>0$ is the premium income rate and $S(t)$ is the cumulated claim amount up to time $t .\{S(t), t \geq 0\}$ is a compound Poisson process, i.e.

$$
S(t)=\sum_{i=1}^{N(t)} X_{i}, \quad t \geq 0,
$$

where $\{N(t), t \geq 0\}$ is a Poisson process, with parameter $\lambda$, which counts the claim occurrences until time $t$, and $\left\{X_{i}, i=1,2, \ldots\right\}$ are the successive claim amounts which are represented by non-negative independent identically distributed random variables. Let $\left\{V_{i}, i=1,2, \ldots\right\}$ be the interarrival times of successive claims. By construction, these random variables are independent with a common exponential distribution. Moreover, the inter-occurrence times are assumed to be independent of the claim amounts.

A statistics of great interest is the probability of (non-)ruin over any fixed horizon of finite length. Denote by $\psi(u, t)$ the probability of ruin before time $t, t \geq 0$, for initial reserves $u$ :

$$
\psi(u, t)=P(\exists s \in[0, t], R(s)<0 \mid R(0)=u), \quad u, t \geq 0 .
$$

Different methods have been proposed to evaluate $\psi(u, t)$; see, e.g., Lefèvre and Loisel (2008).

Recently, much research has been devoted to the evaluation of ruin probabilities, over finite or infinite horizon, when some independence and stationarity assumptions of the model are relaxed. A number of references will be mentioned later in the section (the list being non-exhaustive, of course).

In practice, the independence assumptions on, and between, the sequences $\left\{X_{i}\right\}$ and $\left\{V_{i}\right\}$ may be too unrealistic. Typically, this arises in the case of natural disasters like earthquake (or flooding). The occurrence of an earthquake often increases the probability of by-claims in a near future. If the last earthquake occurred a long time ago, the next earthquake is likely to be more severe. If two earthquakes occur in a short time span, the second one may cause unusual damages like flooding.

This question has already been raised and discussed in the literature. Albrecher and Boxma (2004) consider that the time between two claim occurrences depends on the previous claim amount. Exact expressions for the Laplace transform of the (ultimate) survival probability are derived. In Albrecher and Teugels (2006), the interclaim time 
and the subsequent claim size are dependent through an arbitrary copula structure. Asymptotic results for both the finite and infinite-time ruin probabilities are then derived. Boudreault et al. (2006) consider a particular form of dependence among the interclaim time and the subsequent claim size : if the current interclaim time exceeds a certain threshold, the distribution of the next claim is modified. The defective renewal equation satisfied by the expected Gerber-Shiu discounted penalty function is then obtained. In Meng et al. (2008), the time between two claim occurrences determine the distribution of the next claim. Some exact and approximation results are derived for the survival probability. Ambagaspitiya (2009) determines the ruin probability for two forms of dependence between claim size and occurrence in the Sparre Andersen model. See also the references given in these papers.

The present paper discusses several scenarios of dependence between claim amounts and interclaim arrivals. Such scenarios are motivated by earthquake or flooding-type risks. Our purpose is to provide approximations to the finite-time ruin probabilities, for large initial reserves, when the claim amounts have heavy-tailed distributions. This work can also be seen as a sequel to a recent paper by Biard et al. (2008). Here the claim amount distributions are allowed to depend, to some extent, on the history of the claim arrival process.

\subsection{Basic assumptions and implications}

As in the classical model, claims occur according to a Poisson process $\{N(t), t \geq 0\}$. Let $U_{i}=\sum_{j=1}^{i} V_{j}, i \geq 1$, be the claim arrival times.

Let us turn to the claim amounts $\left\{X_{i}, i \geq 1\right\}$. Firstly, the $X_{i}$ is allowed to be one of two different types of random variables $Y_{i}$ or $Z_{i}$ depending on the behavior of past interarrival times $V_{j}, j \leq i$. Three different models that describe this dependence will be examined.

Secondly, all the severities $Y_{i}$ have the same distribution function (d.f.) $F$, and each vector $\mathbf{Y}^{(j)}=\left(Y_{1}, \ldots, Y_{j}\right), j \geq 1$, is of multivariate regular variation of index $-\alpha$ with $\alpha>0$, i.e. there exists a $\theta \in \mathbf{S}^{j-1}$, where $\mathbf{S}^{j-1}$ is the unit sphere with respect to a norm $|\bullet|$, such that

$$
\frac{P\left(\left|\mathbf{Y}^{(j)}\right|>t x, \mathbf{Y}^{(j)} /\left|\mathbf{Y}^{(j)}\right| \in \bullet\right)}{P\left(\left|\mathbf{Y}^{(j)}\right|>x\right)} \stackrel{v}{\rightarrow} t^{-\alpha} P_{\mathbf{S}^{j-1}}(\theta \in \bullet),
$$

where $\stackrel{v}{\rightarrow}$ denotes vague convergence on $\mathbf{S}^{j-1}$ (see e.g. Resnick (2004) and Basrak et al. (2002)). Analogously, the amounts $Z_{i}$ too have a common distribution function $G$, and each vector $\mathbf{Z}^{(j)}=\left(Z_{1}, \ldots, Z_{j}\right), j \geq 1$, is again of multivariate regularly varying but of index $-\beta$ with $\beta>0$. The sequences $\left\{Y_{i}, i \geq 1\right\},\left\{Z_{i}, i \geq 1\right\}$ and $\left\{V_{i}, i \geq 1\right\}$ are independent of each other.

A key tool in the analysis will be the following well-known result (see e.g. Barbe et al. (2006)). If $\left(Y_{1}, \ldots, Y_{j}\right)$ is regularly varying of index $-\alpha$ with common marginal d.f. $F$, then the right tail of the partial sum $S_{j}=Y_{1}+\ldots+Y_{j}$ can be approximated as

$$
P\left(Y_{1}+\ldots+Y_{j}>x\right) \sim q_{j, \alpha} \bar{F}(x) \text { for large } x,
$$


where $\sim$ means that the ratio tends to 1 as $x \rightarrow \infty$. The parameter $q_{j, \alpha}$ in (1.4) depends on $j, \alpha$ and on the dependence structure inside the vector $\left(Y_{1}, \ldots, Y_{j}\right)$.

Table 1 provides examples of $q_{j, \alpha}$ for some classical multivariate copulas (see Nelsen (2006) for a nice introduction to copulas). The independent copula corresponds to the special case where the $Y_{i}, 1 \leq i \leq j$, are independent. The Fréchet upper bound represents the case where the $Y_{i}$ 's are comonotonic. The non-degenerate Gaussian copula is the case where the dependence between the $Y_{i}{ }^{\prime} \mathrm{s}$ is drawn from a multivariate Gaussian distribution with a correlation matrix with coefficients strictly than \pm 1 outside of the diagonal. A copula introduced in Biard et al. (2008), denoted BBL in the sequel, is built as follows :

$$
Y_{i}=I_{i} W_{0}+\left(1-I_{i}\right) W_{i}, \quad 1 \leq i \leq j,
$$

where $W_{i}, 0 \leq i \leq j$, are i.i.d. random variables, and $I_{i}, 1 \leq i \leq j$, are i.i.d. Bernoulli random variables with parameter $p$, these two sequences being mutually independent.

\begin{tabular}{|l|c|}
\hline Special copulas & $q_{j, \alpha}$ \\
\hline Independent & $j$ \\
Fréchet upper bound & $j^{\alpha}$ \\
Non-degenerate Gaussian & $j$ \\
BLL & $\sum_{i=0}^{j}\left(\begin{array}{c}j \\
i\end{array}\right) p^{i}(1-p)^{j-i}\left(j-i+i^{\alpha}\right)$ \\
\hline
\end{tabular}

ТАв. 1 - Values of $q_{j, \alpha}$ in (1.4) for different copulas.

Let us mention that the study of the sum of dependent random variables has received much attention in actuarial sciences. See e.g. Wüthrich (2003), Alink et al. (2004), Alink et al. (2005), Barbe et al. (2006), Albrecher et al. (2006), Biard et al. (2008) and Kortschak and Albrecher (2009), among many others.

The paper is organized as follows. In Section 2, we obtain the asymptotic finite-time ruin probabilities for regularly varying claim sizes in the risk model introduced by Boudreault et al. (2006). In Section 3, we derive such ruin probabilities in two other risk models, for earthquake or flooding-type risks, that take consecutive gauge-loading effects into account. The methods of proof will rely on the approximation (1.4) and the analysis of spacings in a conditioned Poisson process. Finally, some numerical illustrations are presented in Section 4.

\section{Direct effects of each interclaim arrival}

Our starting point is the model of Boudreault et al. (2006) where claim amounts are of two different types depending on the length of the previous interclaim arrival. Hereafter, we are going to assume that the claim amounts may be dependent and they have heavy tailed distributions.

Specifically, if an interarrival period $V_{i}$ is larger than a fixed threshold $\tau$, then the next claim amount $X_{i}$ is given by a random variable $Y_{i}$, and if not, $X_{i}$ corresponds 
to another random variable $Z_{i}$. As stipulated before, each vector $\mathbf{Y}^{(j)}=\left(Y_{1}, \ldots, Y_{j}\right)$ is of multivariate regular variation of index $-\alpha$ and common d.f. $F$, while each vector $\mathbf{Z}^{(j)}=\left(Z_{1}, \ldots, Z_{j}\right)$ is of multivariate regularly varying of index $-\beta$ and common d.f. $G$.

For instance, for earthquake-type risks, one would expect that $\alpha<\beta$ : the longer a period without any earthquake, more serious will be the next earthquake. For flooding risks, the inverse situation where $\alpha>\beta$ seems to be quite plausible.

Consider the random variable $M(t, \tau)$ that gives the number of spacings of the Poisson process $\{N(s), 0 \leq s \leq t\}$ which are larger than $\tau$. Conditioning by the number of events $N(t)$, define the following.

Let $M(n, t, \tau)$ be the random variable that counts the number, during $(0, t)$, of Poisson spacings which are larger than $\tau$, given that $N(t)=n(\geq 1)$ and $0<\tau<t$.

Proposition 2.1 If $\alpha<\beta$, for $t>0$ and large $u$,

$$
\psi(u, t) \sim\left\{\sum_{n=1}^{\infty} P[N(t)=n] \sum_{j=1}^{\min (n, L t / \tau\rfloor)} P[M(n, t, \tau)=j] q_{j, \alpha}\right\} \bar{F}(u+c t),
$$

while if $\alpha>\beta$, for $t>0$ and large $u$,

$$
\psi(u, t) \sim\left\{\sum_{n=1}^{\infty} P[N(t)=n] \sum_{j=\max (1, n-\lfloor t / \tau\rfloor)}^{n} P[M(n, t, \tau)=n-j] q_{j, \beta}\right\} \bar{G}(u+c t),
$$

with, for $0 \leq j \leq n$,

$$
P[M(n, t, \tau)=j]=\sum_{i=j}^{n}(-1)^{i-j}\left(\begin{array}{c}
n \\
j
\end{array}\right)\left(\begin{array}{c}
n-j \\
i-j
\end{array}\right) P\left(V_{1}^{*}>\frac{\tau}{t}, \ldots, V_{i}^{*}>\frac{\tau}{t}\right),
$$

where

$$
P\left(V_{1}^{*}>v, \ldots, V_{i}^{*}>v\right)= \begin{cases}1, & v \leq 0 \\ (1-i v)^{n}, & 0<v<1 / i \\ 0, & 1 / i \leq v<1\end{cases}
$$

Proof. Consider $S(t)$, the aggregate claim amount (1.2). Following Biard et al. (2008), we know that if $x \mapsto P[S(t)>x]$ is regularly varying, then for large $u$,

$$
\psi(u, t) \sim P[S(t)>u+c t] .
$$

To begin with, we find that

$$
\begin{aligned}
& P[S(t)>x]= \sum_{n=1}^{\infty} P[N(t)=n] P\left(X_{1}+\ldots+X_{n}>x\right) \\
&= \sum_{n=1}^{\infty} P[N(t)=n] \sum_{j=1}^{n} P[M(t, \tau)=j \mid N(t)=n] \\
& P\left[X_{1}+\ldots+X_{n}>x \mid N(t)=n, M(t, \tau)=j\right],
\end{aligned}
$$


and by the model assumptions,

$$
\begin{aligned}
P[S(t)>x]=\sum_{n=1}^{\infty} P[N(t)=n] \sum_{j=1}^{n} P[M(n, t, \tau)=j] \\
P\left(Y_{1}+\ldots+Y_{j}+Z_{1}+\ldots+Z_{n-j}>x\right) .
\end{aligned}
$$

Note that

$$
P[M(n, t, \tau)=j]=0 \text { for } j>t / \tau,
$$

so that the summation over $j$ in (2.7) goes from 1 to $\min (n,\lfloor t / \tau\rfloor)$. Now, suppose that $\alpha<\beta$, so that $G(x)=o(F(x))$ for large $x$. Putting $S_{k}=Y_{1}+\ldots+Y_{k}$ and $T_{k}=Z_{1}+\ldots+Z_{k}$, $k \geq 1$, and using Proposition 1.1 in Cai and Tang (2004) and (1.4), we obtain

$$
\begin{aligned}
P\left(S_{j}+T_{n-j}>x\right) & \sim P\left(S_{j}>x\right)+P\left(T_{n-j}>x\right) \\
& \sim P\left(S_{j}>x\right) \sim q_{j, \alpha} \bar{F}(x), \quad 1 \leq j \leq n .
\end{aligned}
$$

Inserting (2.7) in (2.6) then yields

$$
P[S(t)>x] \sim\left\{\sum_{n=1}^{\infty} P[N(t)=n] \sum_{j=1}^{\min (n, L t / \tau\rfloor)} P[M(n, t, \tau)=j] q_{j, \alpha}\right\} \bar{F}(x),
$$

that is, the function $P[S(t)>x]$ is regularly varying with parameter $\alpha$. Therefore, (2.5) is applicable and gives the announced result (2.1). The approximation (2.2) is derived in a similar way.

Now, it remains to evaluate the distribution of the variable $M(n, t, \tau)$. As $\{N(t), t \geq 0\}$ is a Poisson process, it is well-known that given $N(t)=n$, the claim instants $U_{1}, \ldots, U_{n}$ are distributed as the order statistics of $n$ uniform random variables on $[0, t]$, and the interclaim arrivals $V_{1}, \ldots, V_{n}$ (i.e. the associated spacings) are exchangeable random variables such that the p.d.f. of the vector $\left(V_{1}^{*}, \ldots, V_{i}^{*}\right)$, where $V_{i}^{*}=V_{i} / t, 1 \leq i \leq n$, is given by

$$
\begin{aligned}
f\left(v_{1}, \ldots, v_{i}\right)=\frac{n !}{(n-i) !}\left(1-v_{1}-\ldots-v_{i}\right)^{n-i} \\
\quad \text { for } v_{1}, \ldots, v_{i} \geq 0, v_{1}+\ldots+v_{i} \leq 1 ;
\end{aligned}
$$

see e.g. David and Nagaraja (2003), Sec. 6.4. Consequently, whenever $v_{1}, \ldots, v_{j} \geq 0$ with $v_{1}+\ldots+v_{i} \leq 1$

$$
P\left(V_{1}^{*}>v_{1}, \ldots, V_{i}^{*}>v_{i}\right)=\left(1-v_{1}-\ldots-v_{i}\right)^{n} .
$$

In particular, choosing $v_{i}=\tau / t=v$ for all $i$ in (2.8), we find that $P\left(V_{1}>\tau, \ldots, V_{i}>\tau\right)$ is provided by the formula (2.4) above. Let us consider the event that exactly $j$ interclaim arrivals are larger than $\tau$, given that $N(t)=n$, with $1 \leq j \leq n$. Following e.g. David and 
Nagaraja (2003) (p. 129-130), we then get

$$
\begin{aligned}
P[M(n, t, \tau)=j] & =\frac{1}{j !} \sum_{l=0}^{n-j} \frac{(-1)^{l}}{l !}(j+l) ! \sum_{i_{1}<\cdots<i_{j+l}} P\left(V_{i_{1}}>\tau, \ldots, V_{i_{j+l}}>\tau \mid N(t)=n\right) \\
& =\frac{1}{j !} \sum_{l=0}^{n-j} \frac{(-1)^{l}}{l !}(j+l) !\left(\begin{array}{c}
n \\
j+l
\end{array}\right) P\left(V_{1}>\tau, \ldots, V_{j+l}>\tau \mid N(t)=n\right) \\
& =\sum_{i=j}^{n}(-1)^{i-j} \frac{n !}{j !(i-j) !(n-i) !} P\left(V_{1}^{*}>v, \ldots, V_{i}^{*}>v\right),
\end{aligned}
$$

with $v=\tau / t . \diamond$

\section{Consecutive gauge-loading effects}

In this section, we want to incorporate in the risk model the observation that several consecutive claims with large, or small, interoccurrence times are more susceptible to be followed by severe damages. By comparison with the previous model, it will be necessary this time to take (part of) the history of the claim arrival process into account. So, a gauge will be used to register the large, or small, interclaim arrivals ; initially, the gauge is empty.

\subsection{Earthquake-type phenomenon}

Roughly speaking, for a risk of earthquake-type, the severity of a catastrophe is expected to be more important if the latest catastrophes occur a long time ago. In this sense, let us assume that if $k$ consecutive interclaim arrivals are larger than $\tau$, then a more dangerous catastrophe may arise. Moreover, just after, the gauge is put at level 0 . Damages caused by standard earthquakes give multivariate regular varying vectors $\mathbf{Z}^{(j)}$ of index $-\beta$, while the more severe form regular varying vectors $\mathbf{Y}^{(j)}$ with index $-\alpha$ and common d.f. $F$.

Let $M_{+}(n, k, t, \tau)$ be the random variable that counts the number of sequences, during $(0, t)$, of $k$ consecutive Poisson spacings which are larger than $\tau$, given that $N(t)=n$ $(\geq 1)$.

Proposition 3.1 If $\alpha<\beta$, for $t>0$ and large $u$ :

$$
\psi(u, t) \sim\left\{\sum_{n=1}^{\infty} P[N(t)=n] \sum_{j=1}^{\min (\lfloor n / k\rfloor\rfloor t / k \tau\rfloor)} P\left[M_{+}(n, k, t, \tau)=j\right] q_{j, \alpha}\right\} \bar{F}(u+c t),
$$


with, for $0 \leq j \leq \min (\lfloor n / k\rfloor,\lfloor t / k \tau\rfloor)$,

$$
\begin{aligned}
P\left[M_{+}(n, k, t, \tau)=j\right]=\sum_{i=0}^{k-1} \sum_{x_{1}, \ldots, x_{k}}\left(\begin{array}{c}
x_{1}+\cdots+x_{k}+j \\
x_{1}, \ldots, x_{k}, j
\end{array}\right) \\
P\left[M(n, t, \tau)=n-x_{1}-\cdots-x_{k}\right] /\left(\begin{array}{c}
n \\
x_{1}+\cdots+x_{k}
\end{array}\right),
\end{aligned}
$$

where the inner summation is over all nonnegative integers $x_{1}, \ldots, x_{k}$ such that $x_{1}+2 x_{2}+\cdots+$ $k x_{k}=n-i-k j$ and $x_{2}+2 x_{3}+\ldots+(k-1) x_{k} \leq\lfloor t / \tau\rfloor-i-k j$.

Proof. It suffices to substitute $M_{+}(n, k, t, \tau)$ for $M(n, t, \tau)$ in the proof of Proposition 2.1. By adapting and combining (2.6) and (2.7), we then find that

$$
P[S(t)>x]=\sum_{n=1}^{\infty} P[N(t)=n] \sum_{j=1}^{n} P\left[M_{+}(n, t, \tau)=j\right] q_{j, \alpha} \bar{F}(x),
$$

which leads again to the desired approximation (3.1).

One has now to determine the p.m.f. of the random variable $M_{+}(n, k, t, \tau)$. This is closely related to the evaluation of the so-called binomial distributions of order $k$ (see e.g. the book by Balakrishnan and Koutras (2002)). Remember that the p.m.f. of $M(n, t, \tau)$ is given in Proposition 2.2. Denote by $S$ the event [a Poisson spacing until time $t$ is larger than $\tau$, given that $N(t)=n$ ], and let $F$ be the complementary event. Following Philippou and Makri (1986), we observe that a typical element of the event $\left[M_{+}(n, k, t, \tau)=j\right]$ consists in an arrangement of the form

$$
a_{1} a_{2} \ldots a_{x_{1}+\ldots+x_{k}+j} \underbrace{S S \ldots S}_{i} \text {, with } 0 \leq i \leq k-1,
$$

where $x_{1}$ of the $a^{\prime}$ s are $F, x_{2}$ of the $a^{\prime}$ s are $S F, \ldots, x_{k}$ of the $a^{\prime}$ s are $S S \ldots S F$ and $j$ of the $a^{\prime}$ s are $\underbrace{S S \ldots S}_{k}$. Note that these $x_{k}$ are subject to the constraint $x_{1}+2 x_{2}+\ldots+k x_{k}+k j+i=n$. Moreover, by construction of the model, the number of $S$ has to be smaller than $\lfloor t / \tau\rfloor$, so that $x_{2}+2 x_{3}+(k-1) x_{k}+k j+i \leq\lfloor t / \tau\rfloor$. Now, such arrangements are in number

$$
\left(\begin{array}{c}
x_{1}+\ldots+x_{k}+j \\
x_{1}, \ldots, x_{k}, j
\end{array}\right)
$$

and each of them has probability

$$
P\left[M(n, t, \tau)=n-x_{1}-\cdots-x_{k}\right] /\left(\begin{array}{c}
n \\
x_{1}+\cdots+x_{k}
\end{array}\right),
$$

hence the formula (3.2). $\diamond$ 


\subsection{Flooding-type phenomenon}

For a risk of flooding-type, a close succession of claims is expected to be followed by a more important catastrophe. So, we here assume that if $k$ consecutive interclaim arrivals are smaller than $\tau$, then the next claim may be more severe. Moreover, the gauge becomes empty once an interoccurrence time is larger than $\tau$ (it corresponds to a reconstruction time). In case of standard floods, damages form multivariate regular varying vectors $\mathbf{Y}^{(j)}$ of index $-\alpha$, while the more severe give regular varying vectors $\mathbf{Z}^{(j)}$ with index $-\beta$ and common d.f. $G$.

Let $M_{-}(n, k, t, \tau)$ be the random variable that counts the number of sequences, during $(0, t)$, of at least $k$ consecutive Poisson spacings which are smaller than $\tau$, given that $N(t)=n(\geq 1)$.

Proposition 3.2 If $\alpha>\beta$, for $t>0$ and large $u$ :

$$
\psi(u, t) \sim\left\{\sum_{n=1}^{\infty} P[N(t)=n] \sum_{j=1}^{\min (\lfloor(n+1) /(k+1)],\lfloor t /(\tau-1)\rfloor)} P\left[M_{-}(n, k, t, \tau)=j\right] q_{j, \beta}\right\} \bar{G}(u+c t),
$$

with, for $0 \leq j \leq \min (\lfloor(n+1) /(k+1)\rfloor,\lfloor t /(\tau-1)\rfloor)$,

$$
\begin{array}{r}
P\left[M_{-}(n, k, t, \tau)=j\right]=\sum_{i=0}^{n} \sum_{x_{1}, \ldots, x_{n}}\left(\begin{array}{c}
x_{1}+\cdots+x_{n} \\
x_{1}, \ldots, x_{n}
\end{array}\right) \\
P\left[M(n, t, \tau)=x_{1}+\cdots+x_{n}\right] /\left(\begin{array}{c}
n \\
x_{1}+\cdots+x_{n}
\end{array}\right),
\end{array}
$$

where the inner summation is over all nonnegative integers $x_{1}, \ldots, x_{n}$ such that $x_{1}+2 x_{2}+\cdots+$ $n x_{n}=n-i, x_{k+1}+\ldots+x_{n}+\mathbb{1}_{\{i \geq k\}}=j$ and $x_{1}+\ldots+x_{n} \leq\lfloor t / \tau\rfloor$.

Proof. Substituting this time $M_{-}(n, k, t, \tau)$ for $M(n, t, \tau)$ in the proof of Proposition 2.1 allows us to derive similarly the desired approximation (3.3).

To determine the p.m.f. of the random variable $M_{-}(n, k, t, \tau)$, one still may argue as for $M_{+}(n, k, t, \tau)$. Let $S$ be here the event [a Poisson spacing until time $t$ is smaller than $\tau$, given that $N(t)=n]$, and let $F$ be the complementary event. An element of the event $\left[M_{-}(n, k, t, \tau)=j\right]$ is an arrangement of the form

$$
a_{1} a_{2} \ldots a_{x_{1}+\ldots+x_{n}} \underbrace{S S \ldots S}_{i} \text {, with } 0 \leq i \leq n,
$$

where $x_{1}$ of the $a^{\prime}$ s are $F, x_{2}$ of the $a^{\prime}$ s are $S F, \ldots, x_{n}$ of the $a^{\prime}$ s are $\underbrace{S S \ldots S}_{n-1} F$, with the constraints $x_{1}+2 x_{2}+\ldots+n x_{n}+i=n$ and $x_{k+1}+\ldots+x_{n}+\mathbb{1}_{\{i \geq k\}}=j$. In addition, as the number of spacings larger than $\tau$ is smaller than $\lfloor t / \tau\rfloor$, one has $x_{1}+\ldots+x_{n} \leq\lfloor t / \tau\rfloor$. Now, the number of such arrangements is

$$
\left(\begin{array}{c}
x_{1}+\ldots+x_{n} \\
x_{1}, \ldots, x_{n}
\end{array}\right)
$$


each of them having probability

$$
P\left[M(n, t, \tau)=x_{1}+\ldots+x_{n}\right] /\left(\begin{array}{c}
n \\
x_{1}+\cdots+x_{n}
\end{array}\right),
$$

so that the formula (3.4) then follows. $\diamond$

\section{Numerical analysis}

A central step of the numerical analysis is the computation of the p.m.f. of the random variables $M(n, t, \tau), M_{+}(n, k, t, \tau)$ and $M_{-}(n, k, t, \tau)$. For that, one can use the exact formulas obtained before. Another possible way is to proceed by recursion. A recursive method is provided in the Appendix for the variables $M_{+}$and $M_{-}$(it is not simpler for $M$ ). Both methods have their own advantages and drawbacks.

- The exact formulas are easier to implement but the computation is longer.

- The recursive method works faster but implementation is fastidious.

Hereafter, we have chosen to work with the exact formulas because we aim to see the impact of various dependence parameters on the asymptotic ruin probabilities. Someone who wants to investigate larger time horizons for example, might prefer to follow the recursive method.

\subsection{Impact of a dependence between claim amounts}

In this first part, we consider asymptotic ruin probabilities $\psi(u, t)$ for different values of $q_{k, \alpha}$ (see Table 1). In order to have a spectrum of dependence, the parameter $p$ (called "dependence parameter") in the BLL copula model is allowed to vary from 0 to 1 with step 0.01 . When the dependence parameter equals 0 we have the independence case, and when it equals 1 we obtain the Fréchet upper bound case. The other parameters are :

\begin{tabular}{c|c|c|c}
$u$ & $c$ & $\lambda$ & Distribution of the riskier claim amount \\
\hline $1,000,000$ & 10 & 0.1 & Pareto law with parameter $\alpha$
\end{tabular}

For the model of Section 2, we examine the case where $\alpha<\beta$. For $t=10$ and for both $\alpha=0.5$ and $\alpha=3$, two cases are investigated, one with $\tau=1$ and the other with $\tau=2$. Each case is plotted first separately and then in a same graph. When $\alpha=0.5$ (Figure 1) the asymptotic ruin probability is a decreasing function of the dependence parameter $p$. It is the opposite when $\alpha=3$ (Figure 2).

For the model of Subsection 3.1, with $k=3$, the same phenomenon is observed (see Figure 3 for $\alpha=0.5$ and Figure 4 for $\alpha=3$ ). 

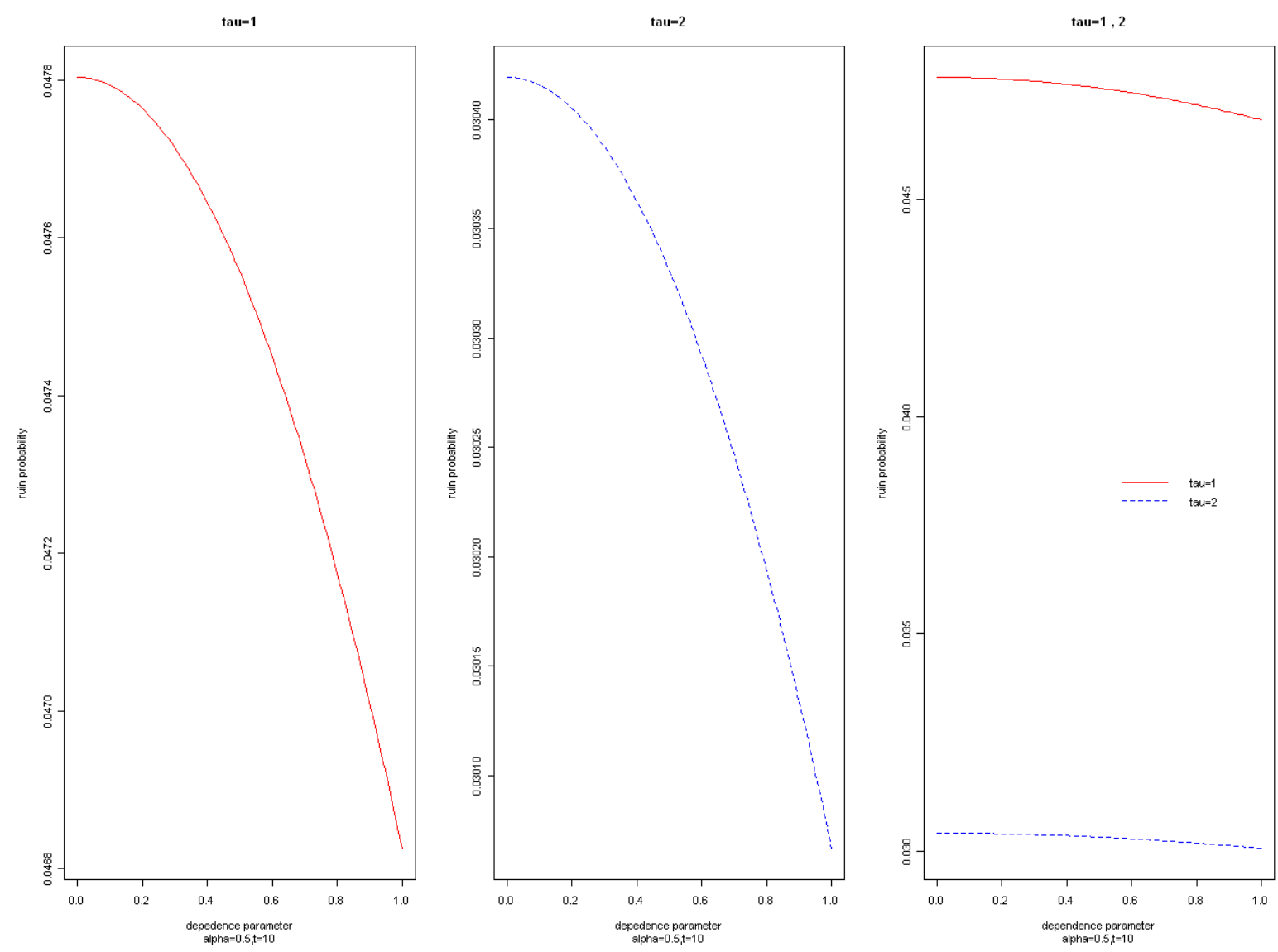

FIG. 1 - Asymptotic ruin probability for the model of Section 2 as a function of the dependence parameter $p$ in the BLL copula when $\alpha=0.5$ and $t=10$.

\subsection{Impact of a dependence between interclaim arrivals and claim amounts}

In this second part, we examine the effect of two parameters on the asymptotic ruin probabilities : $\tau$ for the model of Section 2 and $\tau$ and $k$ for the model of Subsection 3.2. Let us choose $\alpha=3$, for instance. For each case, we consider two dependence parameters $p=0.4$ and $p=0.8$; these are plotted separately and the relative difference is then plotted in a third graph.

For the model of Section 2 with $t=10$, we obtain that as expected, the asymptotic ruin probability is a decreasing function of $\tau$ (see Figure 5).

For the model of Subsection 3.1 with $t=20$, the asymptotic ruin probability is a decreasing function of both $\tau$ and $k$ (see Figures 6 and 7). 

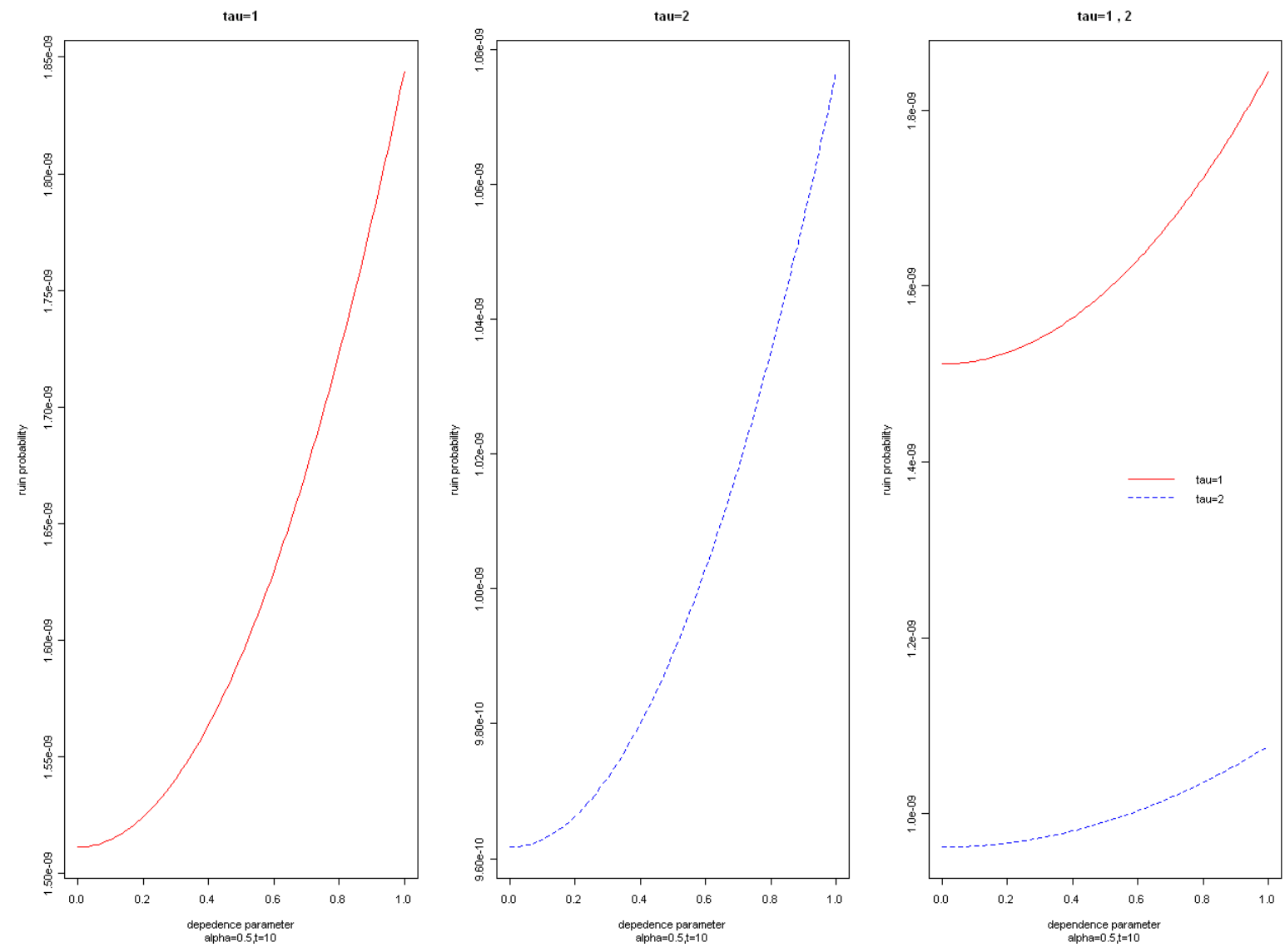

FIG. 2 - Asymptotic ruin probability for the model of Section 2 as a function of the dependence parameter $p$ in the BLL copula when $\alpha=3$ and $t=10$. 

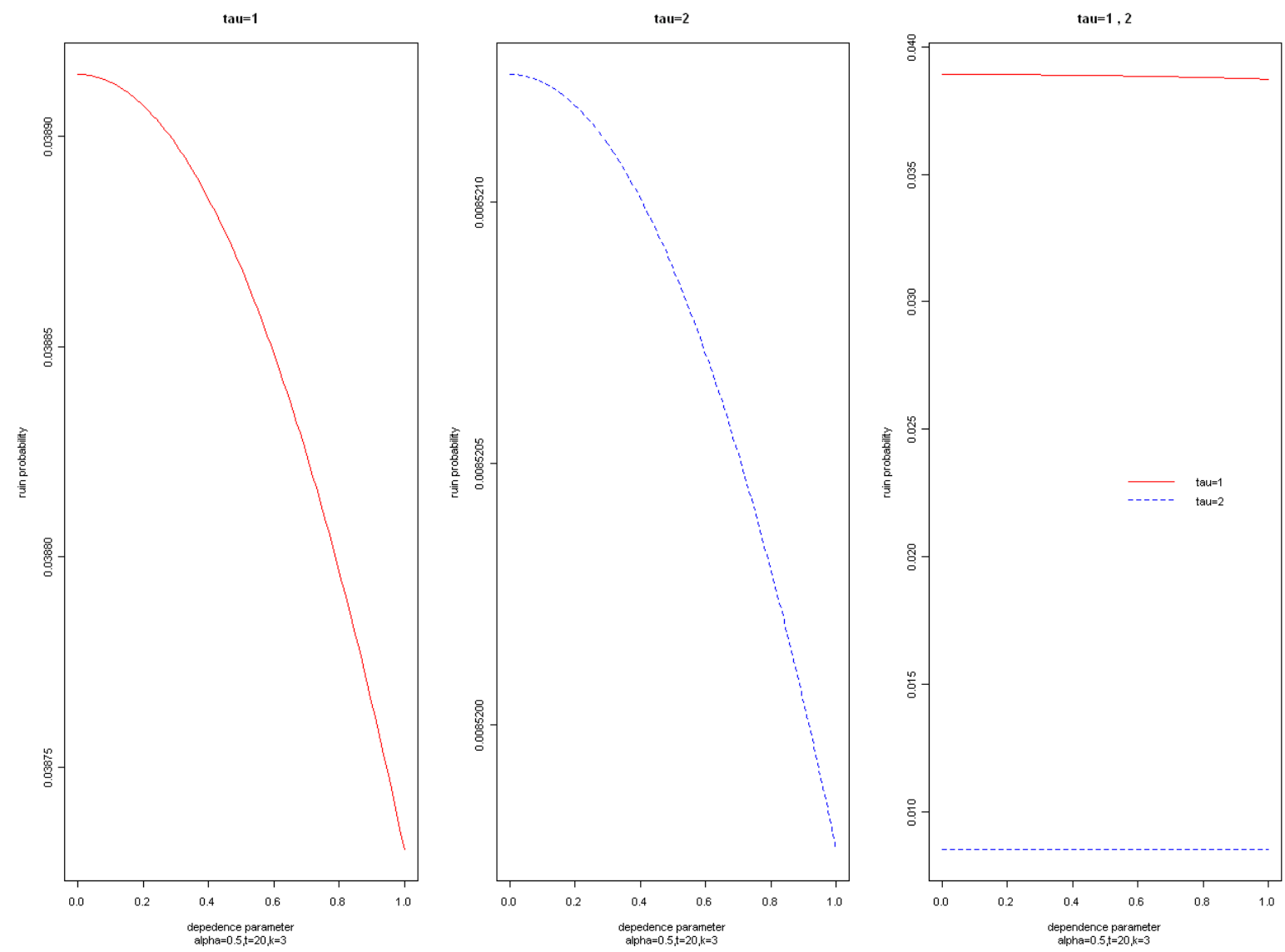

FIG. 3 - Asymptotic ruin probability for the model of Subsection 3.1 as a function of the dependence parameter $p$ in the BLL copula when $\alpha=0.5$ and $t=20$. 

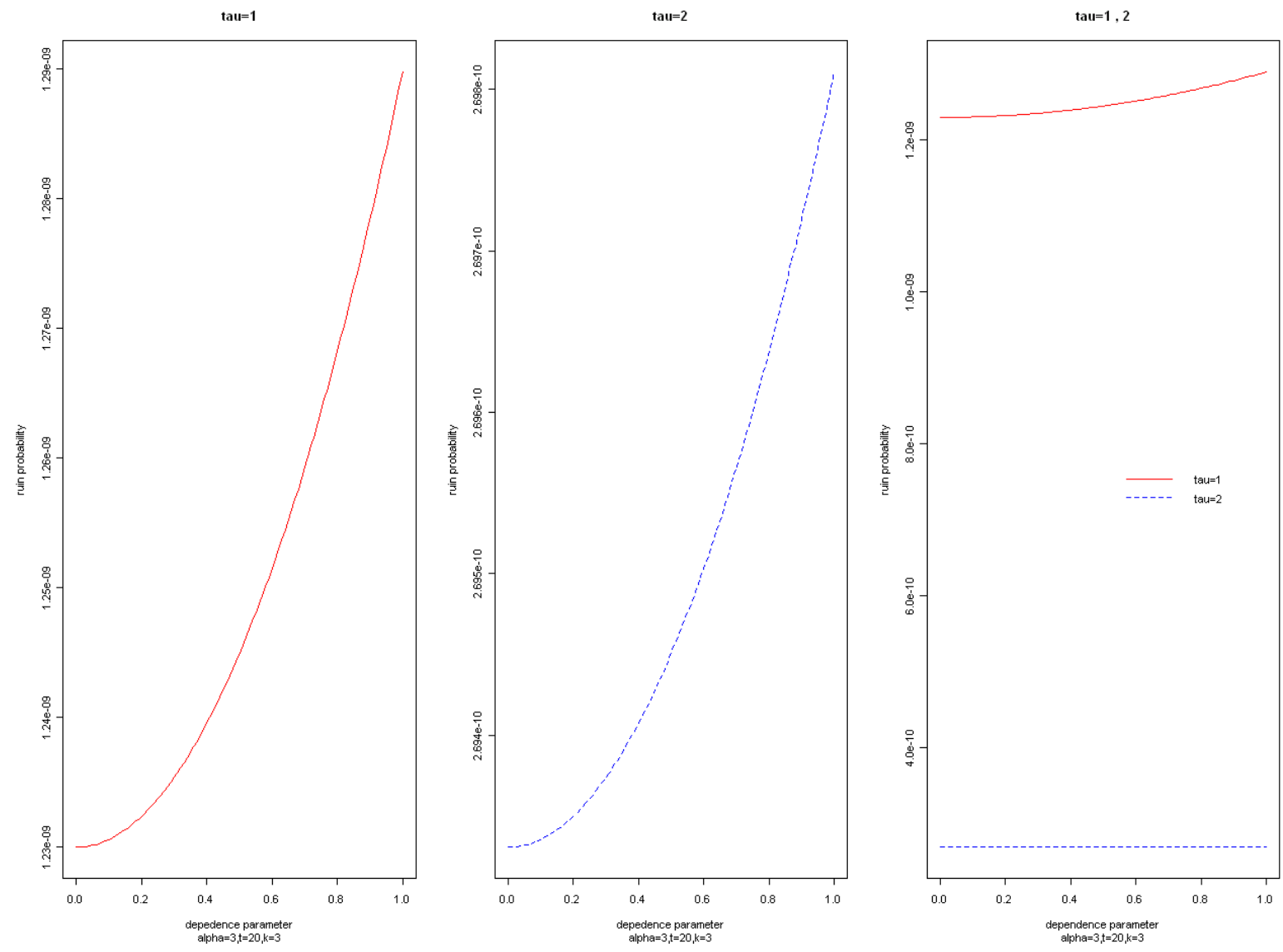

FIG. 4 - Asymptotic ruin probability for the model of Subsection 3.1 as a function of the dependence parameter $p$ in the BLL copula when $\alpha=3$ and $t=20$. 

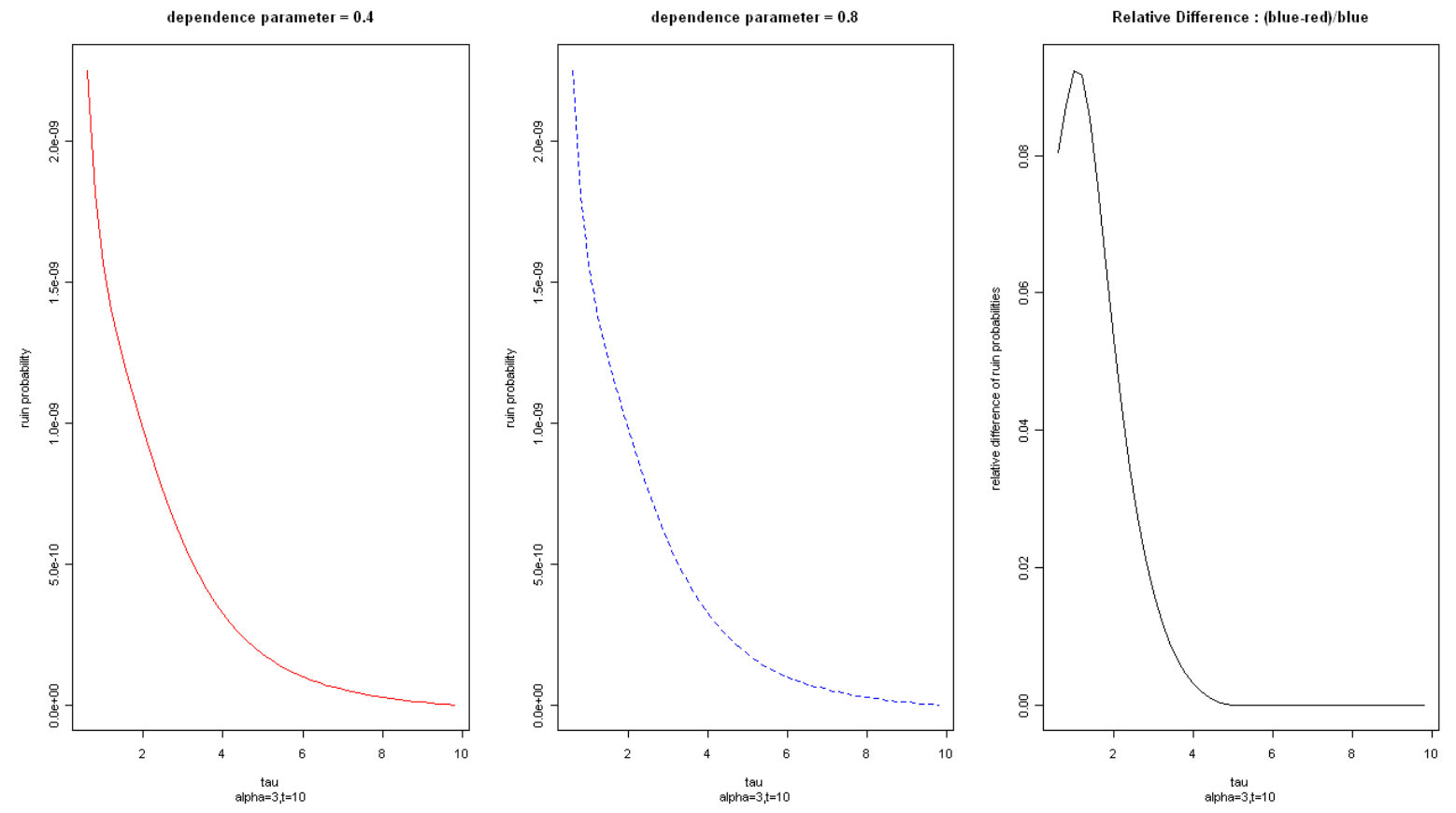

FIG. 5 - Asymptotic ruin probability for the model of Section 2 as a function of $\tau$ when $\alpha=3$ and $t=10$.
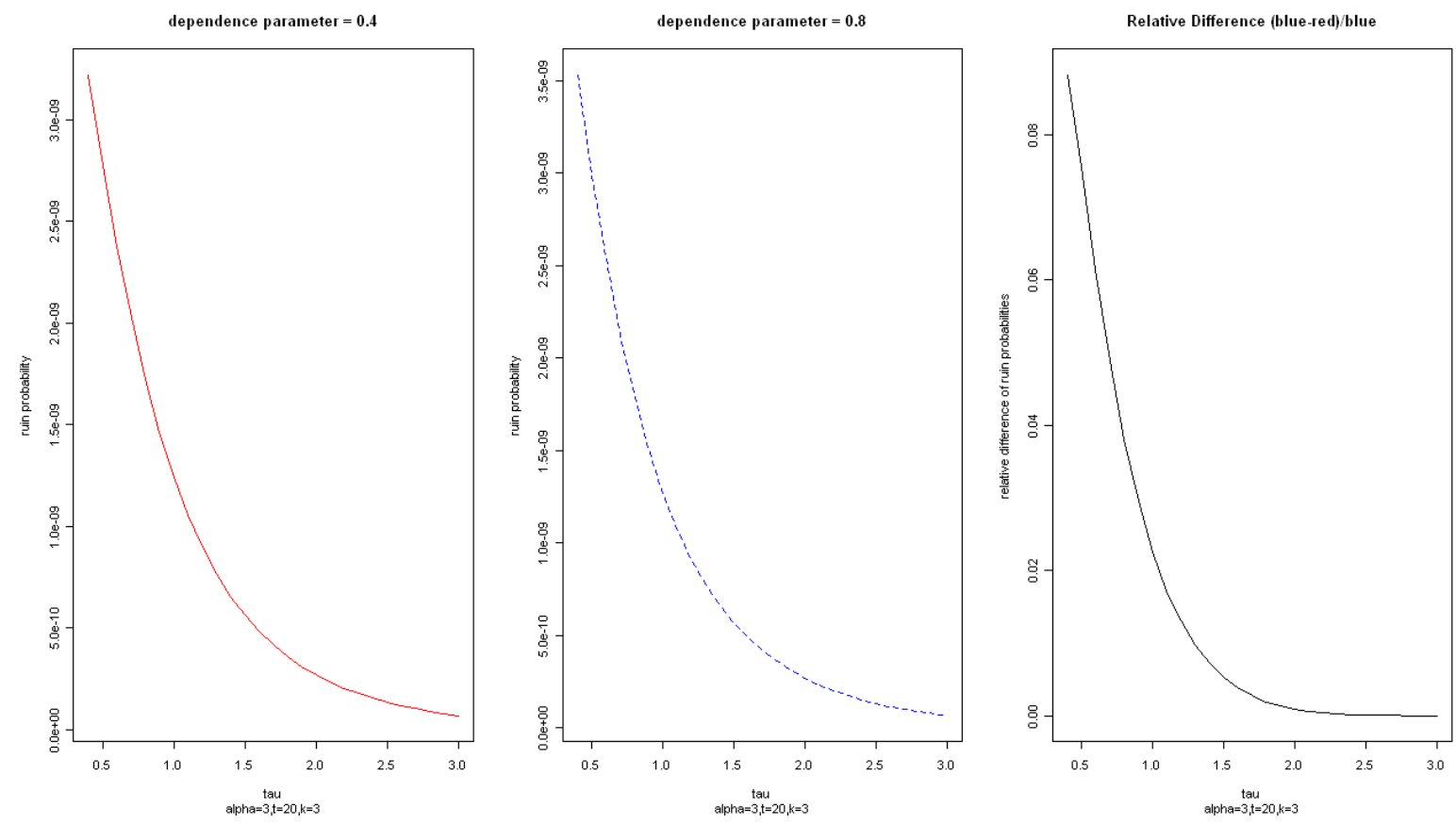

Fig. 6 - Asymptotic ruin probability for the model of Subsection 3.1 as a function of $\tau$ when $\alpha=3$ and $t=20$. 

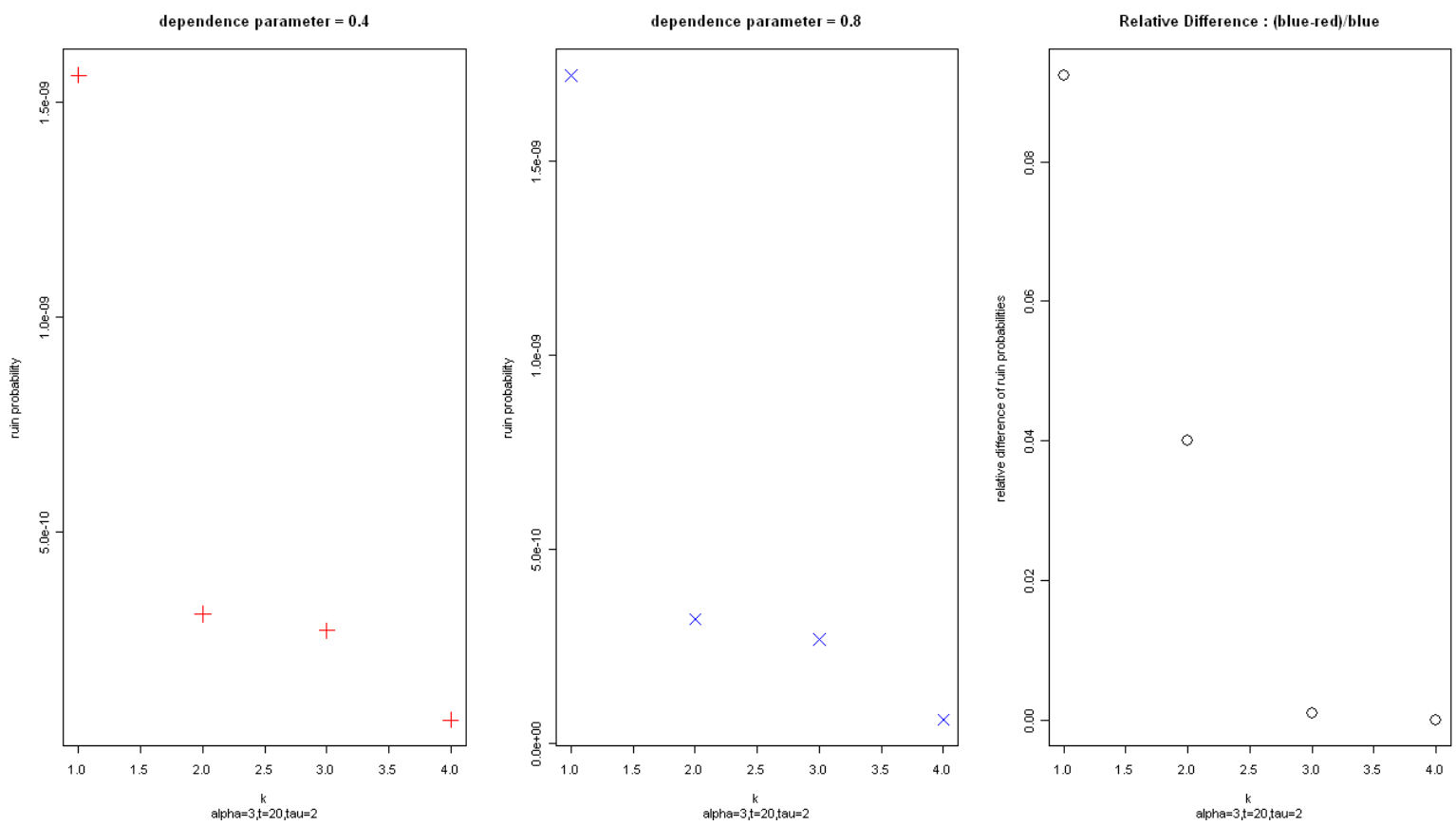

Fig. 7 - Asymptotic ruin probability for the model of Subsection 3.1 as a function of $k$ when $\alpha=3$ and $t=20$. 


\section{Appendix}

We present in this section a recursive method to compute the p.m.f. of $M_{+}(n, k, t, \tau)$ and $M_{-}(n, k, t, \tau)$. The method of proof is directly inspired from Makri and Philippou (2005) (Theorem 4.1).

Proposition 5.1 (a) For $n \geq k+1$ and $1 \leq j \leq \min (\lfloor n / k\rfloor,\lfloor t /(k \tau)\rfloor)$,

$$
\begin{gathered}
P\left[M_{+}(n, k, t, \tau)=j\right]=p_{n, n}(t) \delta_{j, L n / k\rfloor} \\
+\frac{1}{t} \frac{p_{0, n}(t)}{B(1, n)} \int_{0}^{\tau} P\left[M_{+}(n-1, k, t-s, \tau)=j\right]\left(1-\frac{s}{t}\right)^{n} d s \\
+\frac{1}{t} \sum_{l=1}^{k-1} \frac{p_{l, n}(t)}{B(l+1, n-l)} \int_{l \tau}^{t}\left(\frac{s}{t}\right)^{l}\left(1-\frac{s}{t}\right)^{n-l-1} P\left[M_{+}(n-1-l, k, t-s, \tau)=j\right] d s \\
+\frac{1}{t} \sum_{l=k}^{n-1} \frac{p_{l, n}(t)}{B(l+1, n-l)} \int_{l \tau}^{t}\left(\frac{s}{t}\right)^{l}\left(1-\frac{s}{t}\right)^{n-l-1} P\left[M_{+}(n-1-l, k, t-s, \tau)=j-\lfloor l / k\rfloor\right] d s,
\end{gathered}
$$

(b) for $n \geq k+1$,

$$
\begin{aligned}
& P\left[M_{+}(n, k, t, \tau)=0\right]=\frac{1}{t} \frac{p_{0, n}(t)}{B(1, n)} \int_{0}^{\tau} P\left[M_{+}(n-1, k, t-s, \tau)=0\right]\left(1-\frac{s}{t}\right)^{n} d s \\
& +\frac{1}{t} \sum_{l=1}^{k-1} \frac{p_{l, n}(t)}{B(l+1, n-l)} \int_{l \tau}^{t}\left(\frac{s}{t}\right)^{l}\left(1-\frac{s}{t}\right)^{n-l-1} P\left[M_{+}(n-1-l, k, t-s, \tau)=0\right] d s,
\end{aligned}
$$

(c) for $n \geq k+1$ and $j>\min (\lfloor n / k\rfloor,\lfloor t /(k \tau)\rfloor)$,

$$
P\left[M_{+}(n, k, t, \tau)=j\right]=0,
$$

(d) for $n=k$,

$$
\begin{gathered}
P\left[M_{+}(n, k, t, \tau)=0\right]=1-p_{k, k}(t), \\
P\left[M_{+}(n, k, t, \tau)=1\right]=p_{k, k}(t),
\end{gathered}
$$

(e) for $0 \leq n \leq k-1$,

$$
P\left[M_{+}(n, k, t, \tau)=0\right]=1,
$$

where hereabove,

$$
\begin{gathered}
p_{n, n}(t)= \begin{cases}0 & \text { if } n \tau>t, \\
\left(1-\frac{n \tau}{t}\right)^{n} & \text { if } n \tau \leq t,\end{cases} \\
p_{0, n}(t)=1-p_{n, n}(t),
\end{gathered}
$$

and for $1 \leq l \leq n-1$,

$$
p_{l, n}(t)= \begin{cases}0 & \text { if } l \tau>t, \\ \left(1-\frac{l \tau}{t}\right)^{n}-\left(1-\frac{(l+1) \tau}{t}\right)^{n} & \text { if }(l+1) \tau \leq t, \\ \left(1-\frac{l \tau}{t}\right)^{n} & \text { if } l \tau \leq t<(l+1) \tau .\end{cases}
$$




\section{Proof.}

(a) Let $n \geq k+1$ and $1 \leq j \leq \min (\lfloor n / k\rfloor,\lfloor t /(k \tau)\rfloor)$. Define, for $l=0, \ldots, n-1$, the event $A_{l}$ $=[l$ interarrival times larger than $\tau$ precede the first interarrival time smaller than $\tau$ in the sequence of $n$ interarrival times] and the event $B=$ [there is no interarrival time smaller than $\tau$ in the sequence of $n$ interarrival times]. Then, we can write that

$$
\left[M_{+}(n, k, t, \tau)=j\right]=\left(\bigcup_{l=0}^{n-1}\left\{\left[M_{+}(n, k, t, \tau)=j\right] \cap A_{l}\right\}\right) \cup\left\{\left[M_{+}(n, k, t, \tau)=j\right] \cap B\right\},
$$

so that we have

$$
P\left[M_{+}(n, k, t, \tau)=j\right]=\sum_{l=0}^{n-1} P\left[M_{+}(n, k, t, \tau)=j \mid A_{l}\right] P\left(A_{l}\right)+P\left[M_{+}(n, k, t, \tau)=j \mid B\right] P(B) .
$$

For $l=0$,

$$
P\left[M_{+}(n, k, t, \tau)=j \mid A_{0}\right]=\int_{0}^{\tau} P\left[M_{+}(n-1, k, t-s, \tau)=j\right] f_{U_{1}}(s) d s .
$$

For $1 \leq l \leq k-1$

$$
P\left[M_{+}(n, k, t, \tau)=j \mid A_{l}\right]=\int_{l \tau}^{t} P\left[M_{+}(n-1-l, k, t-s, \tau)=j\right] f_{U_{l+1}}(s) d s .
$$

For $k \leq l \leq n-1$,

$$
P\left[M_{+}(n, k, t, \tau)=j \mid A_{l}\right]=\int_{l \tau}^{t} P\left[M_{+}(n-1-l, k, t-s, \tau)=j-\lfloor l / k\rfloor\right] f_{U_{l+1}}(s) d s .
$$

And

$$
P\left[M_{+}(n, k, t, \tau)=j \mid B\right]=\delta_{j,\lfloor n / k\rfloor} .
$$

From (2.4), we get, for $1 \leq l \leq n-1$,

$$
\begin{gathered}
P\left(A_{l}\right)= \begin{cases}0 & \text { if } l \tau>t, \\
P\left(V_{1}>\tau, \ldots, V_{l}>\tau\right)-P\left(V_{1}>\tau, \ldots, V_{l+1}>\tau\right) & \text { if }(l+1) \tau \leq t, \\
P\left(V_{1}>\tau, \ldots, V_{l}>\tau\right) & \text { if } \tau \leq t<(l+1) \tau,\end{cases} \\
= \begin{cases}0 & \text { if } l \tau>t, \\
\left(1-\frac{l \tau}{t}\right)^{n}-\left(1-\frac{(l+1) \tau}{t}\right)^{n} & \text { if }(l+1) \tau \leq t, \\
\left(1-\frac{l \tau}{t}\right)^{n} & \text { if } l \tau \leq t<(l+1) \tau,\end{cases} \\
P\left(A_{0}\right)=1-P\left(V_{1}>\tau\right)=1-\left(1-\frac{\tau}{t}\right)^{n},
\end{gathered}
$$

and

$$
\begin{aligned}
P(B) & = \begin{cases}0 & \text { if } n \tau>t, \\
P\left(V_{1}>\tau, \ldots V_{n}>\tau\right) & \text { if } n \tau \leq t,\end{cases} \\
& = \begin{cases}0 & \text { if } n \tau>t, \\
\left(1-\frac{n \tau}{t}\right)^{n} & \text { if } n \tau \leq t .\end{cases}
\end{aligned}
$$


From David and Nagaraja (2003) p. 134-135, we have, for $l=1, \ldots, n$,

$$
f_{U_{l}}(u)=\frac{1}{t} \frac{1}{B(l, n+1-l)}\left(\frac{u}{t}\right)^{l-1}\left(1-\frac{u}{t}\right)^{n-l},
$$

where $B(.,$.$) is the Beta function. So, we deduce part (a) of the proposition.$

(b) When $j=0$ and $n \geq k$, we observe that

$$
\left[M_{+}(n, k, t, \tau)=0\right]=\bigcup_{l=0}^{k-1}\left\{\left[M_{+}(n, k, t, \tau)=0\right] \cap A_{l}\right\},
$$

so that

$$
P\left[M_{+}(n, k, t, \tau)=0\right]=\sum_{l=0}^{k-1} P\left[M_{+}(n, k, t, \tau)=0 \mid A_{l}\right]
$$

For $l=0$,

$$
P\left[M_{+}(n, k, t, \tau)=0 \mid A_{0}\right]=\int_{0}^{\tau} P\left[M_{+}(n-1, k, t-s, \tau)=0\right] f_{U_{1}}(s) d s .
$$

For $1 \leq l \leq k-1$,

$$
P\left[M_{+}(n, k, t, \tau)=0 \mid A_{l}\right]=\int_{l \tau}^{t} P\left[M_{+}(n-1-l, k, t-s, \tau)=0\right] f_{U_{l+1}}(s) d s .
$$

For $l \geq k$,

$$
P\left[M_{+}(n, k, t, \tau)=0 \mid A_{l}\right]=0 .
$$

Thus, we get part (b) of the proposition.

(c) Obvious fact.

(d) For $n=k$,

$$
P\left[M_{+}(n, k, t, \tau)=1\right]=P\left(V_{1}>\tau, V_{2}>\tau, \ldots, V_{k}>\tau\right)=\left(1-\frac{k \tau}{t}\right)^{k},
$$

and

$$
P\left[M_{+}(n, k, t, \tau)=0\right]=1-P\left[M_{+}(n, k, t, \tau)=1\right] .
$$

(e) Obvious fact. $\diamond$

Proposition 5.2 (a) For $n \geq k+1$ and $1 \leq j \leq \min (\lfloor(n+1) /(k+1)\rfloor,\lfloor t /(\tau-1)\rfloor)$,

$$
\begin{gathered}
P\left[M_{-}(n, k, t, \tau)=j\right]=\delta_{j, 1} q_{n, n}(t) \\
+\frac{1}{t} \sum_{l=0}^{k-1} \frac{q_{l, n}(t)}{B(l+1, n-l)} \int_{\tau}^{t}\left(\frac{s}{t}\right)^{l}\left(1-\frac{s}{t}\right)^{n-l-1} P\left[M_{-}(n-1, k, t-s, \tau)=j\right] d s \\
+\frac{1}{t} \sum_{l=k}^{n-1} \frac{q_{l, n}(t)}{B(l+1, n-l)} \int_{\tau}^{t}\left(\frac{s}{t}\right)^{l}\left(1-\frac{s}{t}\right)^{n-l-1} P\left[M_{-}(n-1-l, k, t-s, \tau)=j-1\right] d s,
\end{gathered}
$$


(b) for $n \geq k+1$,

$$
\begin{aligned}
& P\left[M_{-}(n, k, t, \tau)=0\right]=\frac{1}{t} \sum_{l=0}^{k-1} \frac{q_{l, n}(t)}{B(l+1, n-l)} \\
& \int_{l \tau}^{t}\left(\frac{s}{t}\right)^{l}\left(1-\frac{s}{t}\right)^{n-l-1} P\left[M_{-}(n-1-l, k, t-s, \tau)=0\right] d s,
\end{aligned}
$$

(c) for $n \geq k+1$ and $j>\min (\lfloor(n+1) /(k+1)\rfloor$, $\lfloor t /(\tau-1)\rfloor)$,

$$
P\left[M_{-}(n, k, t, \tau)=j\right]=0,
$$

(d) for $n=k$,

$$
\begin{gathered}
P\left[M_{-}(n, k, t, \tau)=0\right]=1-q_{k, k}(t), \\
P\left[M_{-}(n, k, t, \tau)=1\right]=q_{k, k}(t),
\end{gathered}
$$

(e) for $0 \leq n \leq k-1$,

$$
P\left[M_{-}(n, k, t, \tau)=0\right]=1,
$$

where hereabove,

$$
\begin{gathered}
q_{n, n}(t)=1-\left[n\left(1-\frac{\tau}{t}\right)^{n}-\left(\begin{array}{l}
n \\
2
\end{array}\right)\left(1-\frac{2 \tau}{t}\right)^{n}+\ldots+(-1)^{n-1}\left(1-\frac{n \tau}{t}\right)^{n}\right], \\
q_{0, n}(t)=\left(1-\frac{\tau}{t}\right)^{n},
\end{gathered}
$$

and for $1 \leq l \leq n-1$,

$$
\begin{aligned}
q_{l, n}(t)= & {\left[(l+1)\left(1-\frac{\tau}{t}\right)^{n}-\left(\begin{array}{c}
2 \\
l+1
\end{array}\right)\left(1-\frac{2 \tau}{t}\right)^{n}+\ldots+(-1)^{l}\left(1-\frac{(l-1) \tau}{t}\right)^{n}\right] } \\
& -\left[l\left(1-\frac{\tau}{t}\right)^{n}-\left(\begin{array}{l}
2 \\
l
\end{array}\right)\left(1-\frac{2 \tau}{t}\right)^{n}+\ldots+(-1)^{l-1}\left(1-\frac{l \tau}{t}\right)^{n}\right] .
\end{aligned}
$$

Proof. We will follow an analogous argument.

(a) Let $n \geq k-1$ and $1 \leq j \leq \min (\lfloor(n+1) /(k+1)\rfloor,\lfloor t /(\tau-1)\rfloor)$. Define this time, for $l=0, \ldots, n-1, A_{l}=[l$ interarrival times smaller than $\tau$ precede the first interarrival time larger than $\tau$ in the sequence of $n$ interarrival times] and $B=$ [there is no interarrival time larger than $\tau$ in the sequence of $n$ interarrival times]. Then,

$$
\left.\left.\left[M_{-}(n, k, t, \tau)=j\right]=\left(\bigcup_{l=0}^{n-1}\right\}\left[M_{-}(n, k, t, \tau)=j\right] \cap A_{l}\right\}\right) \cup\left\{\left[M_{-}(n, k, t, \tau)=j\right] \cap B\right\},
$$

so that

$$
P\left[M_{-}(n, k, t, \tau)=j\right]=\sum_{l=0}^{n-1} P\left[M_{-}(n, k, t, \tau)=j \mid A_{l}\right] P\left(A_{l}\right)+P\left[M_{-}(n, k, t, \tau)=j \mid B\right] P(B) .
$$


For $0 \leq l \leq k-1$,

$$
P\left[M_{-}(n, k, t, \tau)=j \mid A_{l}\right]=\int_{\tau}^{t-j \tau+1} P\left[M_{-}(n-1, k, t-s, \tau)=j\right] f_{U_{l+1}}(s) d s .
$$

For $k \leq l \leq n-1$,

$$
P\left[M_{-}(n, k, t, \tau)=j \mid A_{l}\right]=\int_{\tau}^{t-j \tau} P\left[M_{-}(n-1-l, k, t-s, \tau)=j-1\right] f_{U_{l+1}}(s) d s .
$$

And

$$
P\left[M_{-}(n, k, t, \tau)=j \mid B\right]=\delta_{j, 1} .
$$

From David and Nagaraja (2003) p. 126, 134-135, we have, for $1 \leq l \leq n-1$,

$$
\begin{aligned}
P\left(A_{l}\right)= & P\left(V_{1} \leq \tau, \ldots, V_{l} \leq \tau\right)-P\left(V_{1} \leq \tau, \ldots, V_{l+1} \leq \tau\right) \\
= & P\left(V_{1} \leq \tau \cup \ldots \cup V_{l+1} \leq \tau\right)-P\left(V_{1} \leq \tau \cup \ldots \cup V_{l} \leq \tau\right) \\
= & {\left[(l+1)\left(1-\frac{\tau}{t}\right)^{n}-\left(\begin{array}{c}
2 \\
l+1
\end{array}\right)\left(1-\frac{2 \tau}{t}\right)^{n}+\ldots+(-1)^{l}\left(1-\frac{(l-1) \tau}{t}\right)^{n}\right] } \\
& -\left[l\left(1-\frac{\tau}{t}\right)^{n}-\left(\begin{array}{l}
2 \\
l
\end{array}\right)\left(1-\frac{2 \tau}{t}\right)^{n}+\ldots+(-1)^{l-1}\left(1-\frac{l \tau}{t}\right)^{n}\right], \\
& P\left(A_{0}\right)=P\left(V_{1}>\tau\right)=\left(1-\frac{\tau}{t}\right)^{n},
\end{aligned}
$$

and

$$
\begin{aligned}
P(B) & =P\left(V_{(n)} \leq \tau\right)=1-P\left(V_{(n)}>\tau\right) \\
& =1-\left[n\left(1-\frac{\tau}{t}\right)^{n}-\left(\begin{array}{l}
n \\
2
\end{array}\right)\left(1-\frac{2 \tau}{t}\right)^{n}+\ldots+(-1)^{n-1}\left(1-\frac{n \tau}{t}\right)^{n}\right] .
\end{aligned}
$$

Using again the expression of $f_{U_{l}}(u), l=1, \ldots, n$, we then obtain part (a) of the proposition.

(b) As for part (b) of Proposition 5.1.

(c) Obvious fact.

(d) For $n=k$,

$$
\begin{aligned}
P\left[M_{-}(n, k, t, \tau)=0\right] & =P\left(V_{(n)}>\tau\right), \\
& =n\left(1-\frac{\tau}{t}\right)^{n}-\left(\begin{array}{l}
n \\
2
\end{array}\right)\left(1-\frac{2 \tau}{t}\right)^{n}+\ldots+(-1)^{n-1}\left(1-\frac{n \tau}{t}\right)^{n},
\end{aligned}
$$

and

$$
P\left[M_{-}(n, k, t, \tau)=1\right]=1-P\left(V_{(n)}>\tau\right) .
$$

(e) Obvious fact. $\diamond$

\section{Acknowledgment}

C. Lefèvre thanks the members of the I.S.F.A for their warm welcome during his visit, and the Banque Nationale de Belgique for a partial support. 


\section{Références}

Albrecher, H., Asmussen, S., and Kortschak, D. (2006). Tail asymptotics for the sum of two heavy-tailed dependent risks. Extremes, 9(2) :107-130.

Albrecher, H. and Boxma, O. J. (2004). A ruin model with dependence between claim sizes and claim intervals. Insurance : Mathematics $\mathcal{E}$ Economics, 35(2) :245-254.

Albrecher, H. and Teugels, J. L. (2006). Exponential behavior in the presence of dependence in risk theory. Journal of Applied Probability, 43(1) :257-273.

Alink, S., Löwe, M., and V. Wüthrich, M. (2004). Diversification of aggregate dependent risks. Insurance Mathematics and Economics, 35(1):77-95.

Alink, S., Löwe, M., and Wüthrich, M. V. (2005). Analysis of the expected shortfall of aggregate dependent risks. Astin Bulletin, 35(1) :25-43.

Ambagaspitiya, R. S. (2009). Ultimate ruin probability in the sparre andersen model with dependent claim sizes and claim occurrence times. Insurance : Mathematics and Economics, 44(3) :464 - 472 .

Balakrishnan, N. and Koutras, M. V. (2002). Runs and scans with applications. Wiley Series in Probability and Statistics. Wiley-Interscience [John Wiley \& Sons], New York.

Barbe, P., Fougères, A.-L., and Genest, C. (2006). On the tail behavior of sums of dependent risks. Astin Bulletin, 36(2) :361-373.

Basrak, B., Davis, R. A., and Mikosch, T. (2002). A characterization of multivariate regular variation. The Annals of Applied Probability, 12(3) :908-920.

Biard, R., Lefèvre, C., and Loisel, S. (2008). Impact of correlation crises in risk theory : Asymptotics of finite-time ruin probabilities for heavy-tailed claim amounts when some independence and stationarity assumptions are relaxed. Insurance: Mathematics and Economics, 43(3):412 - 421.

Boudreault, M., Cossette, H., Landriault, D., and Marceau, E. (2006). On a risk model with dependence between interclaim arrivals and claim sizes. Scandinavian Actuarial Journal, 2006(5) :265-285.

Cai, J. and Tang, Q. (2004). On max-sum equivalence and convolution closure of heavytailed distributions and their applications. Journal of Applied Probability, 41(1):117-130.

David, H. A. and Nagaraja, H. N. (2003). Order statistics. Wiley Series in Probability and Statistics. Wiley-Interscience [John Wiley \& Sons], Hoboken, NJ, third edition.

Kortschak, D. and Albrecher, H. (2009). Asymptotic results for the sum of dependent non-identically distributed random variables. Methodology and Computing in Applied Probability, to appear. 
Lefèvre, C. and Loisel, S. (2008). On finite-time ruin probabilities for classical risk models. Scandinavian Actuarial Journal, $1: 41-60$.

Makri, F. and Philippou, A. (2005). On binomial and circular binomial distributions of order k for l-overlapping success runs of length k. Statistical Papers, 46(3) :411-432.

Meng, Q., Zhang, X., and Guo, J. (2008). On a risk model with dependence between claim sizes and claim intervals. Statistics \& Probability Letters, 78(13) :1727 - 1734.

Nelsen, R. (2006). An Introduction to Copulas. Springer Science+ Business Media, Inc.

Philippou, A. N. and Makri, F. S. (1986). Successes, runs and longest runs. Statistics $\mathcal{E}$ Probability Letters, 4(4): $211-215$.

Resnick, S. (2004). The extremal dependence measure and asymptotic independence. Stochastic Models, 20(2) :205-227.

Wüthrich, M. V. (2003). Asymptotic value-at-risk estimates for sums of dependent random variables. Astin Bulletin, 33(1) :75-92. 